\title{
REFLEXÃO SOBRE A LEGITIMIDADE DAAUTONOMIA DA ENFERMAGEM NO CAMPO DAS PROFISSÕES DE SAÚDE À LUZ DAS IDEIAS DE ELIOT FREIDSON
}

\author{
Reflection on the legitimacy of the autonomy at nursing in the field of the health \\ professions in the light of Eliot Freidson's ideas. \\ Reflexión sobre la legitimidad de la autonomía de las enfermeras en el ámbito de las \\ profesiones de la salud a la luz de las ideas de Eliot Freidson
}

Maria Lígia dos Reis Bellaguarda ${ }^{1}$

Denise Pires ${ }^{4}$
Maria Itayra Padilha

Maria Angélica de Almeida Peres ${ }^{5}$
André de Faria Pereira Neto ${ }^{3}$

\section{RESUMO}

0 estudo teve o objetivo de refletir acerca da Enfermagem como profissão da saúde à luz da teorização de Eliot Freidson para as profissões. Abordaram-se as credenciais para uma ocupação ser reconhecida como profissão, relacionando-as com as características da enfermagem, especialmente no Brasil. Na abordagem freidsoniana destacam-se como credenciais de profissão a expertise, a autonomia e a autorregulação. A enfermagem é analisada enfatizando a sua responsabilidade por uma gama de fazeres realizados com base em conhecimentos científicos, a necessidade de formação específica para o exercício da profissão, a disponibilidade de saberes e 0 reconhecimento do Estado do credenciamento formal de proteção aos membros da profissão. Conclui-se que a enfermagem é uma profissão da saúde, com papel fundamental para a transformação da realidade da saúde e que necessita investir no convencimento da sociedade acerca de sua utilidade enquanto profissão voltada às necessidades de saúde das pessoas.

Palavras-chave: Enfermagem. Autonomia profissional. Sociologia. Prática profissional.

\begin{abstract}
This study aims to reflect about Nursing as a health profession in the light of Eliot Freidson's theory of professions. It approaches the necessary credentials for an occupation to be recognized as a profession, relating them to the nursing's characteristics, with a special focus in Brazil. In Freidson's approach, expertise, autonomy and self-regulation are highlighted as professional credentials. Nursing is analyzed by emphasizing its responsibility for a range of tasks whose performance is based on scientific knowledge, on the need of specific professional training, on the knowledge availability and the State recognition of formal credentials for the professional member's protection. It concludes that Nursing is a health profession with a key role in the health's reality transformation and that investment is needed to convince society about its usefulness as a profession dedicated to people's health needs.
\end{abstract}

Keywords: Nursing. Professional autonomy. Sociology. Professional Practice.

\section{Resumen}

El estudio tiene por objetivo reflexionar sobre la enfermería como profesión del campo de la salud a la luz de las ideas de Eliot Freidson para las profesiones. Se consideran los meritos para que esta actividad sea reconocida como profesión, relacionándolas con las características de la enfermería, en especial en Brasil. En el abordaje Freidsoniano, se resaltan como meritos de la profesión, la excelencia, la autonomía y la auto-regulación. La enfermería es analizada enfatizando sus responsabilidades en una gama de actividades realizadas sobre la base de conocimientos científicos, la necesidad de formación específica para el ejercicio de la profesión, la disponibilidad de conocimientos y el reconocimiento estatal de la titularización formal, como protección a los miembros de la profesión. Se concluye que la enfermería es una profesión de la salud, con un papel fundamental para la transformación y que necesita invertir en ser reconocida por la sociedad sobre su utilidad como profesión dedicada a la salud de las personas.

Palabras clave: Enfermería. Autonomía Profesional. Sociología. Práctica Profesional

\footnotetext{
"Enfermeira. Mestre e Doutoranda do Programa de Pós-Graduação em Enfermagem da UFSC. Bolsista CAPES em doutorado sanduíche na Lawrence"s Bloomberg Faculty of Nursing - University of Toronto. Membro do Grupo de Estudos da História do Conhecimento em Enfermagem e Saúde - GEHCES/UFSC. Florianópolis-SC. Brasil. E-mail: bellaguardaml@gmail.com; ${ }^{2}$ Enfermeira. Doutora em Enfermagem pela Escola Anna Nery, da Universidade Federal do Rio de Janeiro EEAN/UFR. Pósdoutorado na Lawrence Bloomberg University of Toronto, Canadá. Docente do Departamento de Enfermagem e Pós-graduação em Enfermagem da Universidade Federal de Santa Catarina-UFSC. Líder do GEHCES/UFSC. Pesquisadora do CNPq. Florianópolis- SC. Brasil. E-mail: padilha@ccs.com.br; ${ }^{3}$ Historiador, Doutor em Saúde Coletiva (IMS-UERJ/1997), Pesquisador da Casa de Oswaldo Cruz - Fundação Oswaldo Cruz. Rio de Janeiro-RJ. Brasil. E-mail:apereira@fiocruz.br; ${ }^{4}$ Enfermeira. Doutora em Ciências Sociais pela UNICAMP. Pós-doutorado no Coronel Institute, University of Amsterdam, Holanda. Docente do Departamento de Enfermagem e Pósgraduação em Enfermagem da Universidade Federal de Santa Catarina-UFSC. Pesquisadora do Grupo Praxis: trabalho, cidadania, saúde e enfermagem. Pesquisadora CNPq. Conselheira do Coren/SC. Florianópolis-SC. Brasil. E-mail: piresdp@yahoo.com; ${ }^{5}$ Enfermeira. Professora Adjunta da EEAN/ UFRJ, Doutora em História da Enfermagem. Membro da Diretoria Colegiada do Núcleo de Pesquisa de História da Enfermagem Brasileira. Rio de Janeiro-RJ. Brasil. E-mail:aguaonda@uol.com.br
} 


\section{INTRODUÇÃO}

A enfermagem, como as demais profissões da área da saúde, teve, na sua emergência, influência das necessidades assistenciais da população e das demandas dos movimentos político-sociais por saúde, assim como estiveram vinculadas ao Estado e às forças hegemônicas de poder.

Na perspectiva da sociologia das profissões, ${ }^{1: 15}$ profissão é um tipo especial de ocupação "autônoma e autorregulada," e este status é garantido pela sua capacidade de convencer a sociedade que seus membros são "especialmente confiáveis". 0 exercício da autonomia, "como direito legítimo de controlar o seu trabalho", depende da "tolerância e mesmo da proteção do Estado", portanto não são apenas aspectos técnicos, da expertise, que garantem o status de profissão. ${ }^{1: 102} 0$ reconhecimento de uma ocupação como profissão resulta de um processo complexo "mais político e social do que técnico" . 1:101

As duas credenciais centrais formuladas pela sociologia das profissões - "o treinamento prolongado e especializado de um corpo de conhecimentos abstratos" e o desenvolvimento de "um serviço voltado para a coletividade" não são suficientes para definir profissão, é necessária a aprovação/disponibilização de um "conjunto de regras e regulamentos formais corporificados em leis ou a regulamentos e resoluções vinculados a instituições políticas, associações profissionais e organizações educacionais" 1:99 É necessário agregar conhecimento específico e regulação das atividades para garantir a autonomia profissional.

No que diz respeito à Enfermagem, é importante considerar o contexto de sua estruturação como profissão que tem como referência o trabalho de Florence Nightingale na Inglaterra, no século XIX. Com Florence verifica-se o atendimento de uma necessidade social, a produção de conhecimentos para fundamentação da prática, a estruturação de um modelo de formação e a conquista de algumas regras para a regulação do exercício profissional. Cabe considerar, neste contexto, o poder já instituído socialmente pela profissão médica, as próprias questões de gênero, que historicamente permearam 0 desenvolvimento da Enfermagem, sua autonomia no exercício da assistência às necessidades de saúde das pessoas e populações, assim como as credenciais qualificacionais dos membros da profissão.

A origem da profissão de Enfermagem no Brasil se deu em três fases que caracterizaram seu saber-fazer: primeiro, pela realização de tarefas relacionadas aos papéis femininos tradicionais, considerados socialmente inferiores aos papéis masculinos. Segundo, pela realização de procedimentos justificados técnica e cientificamente, garantidos pela regulação profissional e pelo seguimento de pressupostos de educação, na maioria orientados pelo Sistema Nightingaleano. A terceira fase se inicia em 1970, configurando-se pela construção de um corpo de conhecimento específico para a profissão. ${ }^{2}$
0 Sistema Nightingale chegou ao Brasil, via modelo anglo-americano, em 1921, trazendo, além da mística vocacional, as intenções associativas quanto à legalidade da profissão, as preocupações com o papel social da mulher enfermeira e os propósitos de cuidados não somente aos indivíduos doentes, mas principalmente aos sadios. ${ }^{1: 28} \mathrm{~A}$ estruturação da enfermagem como profissão ocorre no contexto da sociedade brasileira agro-exportadora das primeiras décadas do século XX, que necessitava de intervenção do Estado para o controle das grandes epidemias e endemias que assolavam 0 país. ${ }^{3}$

Nas décadas de 1940-1950 no âmbito da saúde, no Brasil, destacou-se a política previdenciária, privilegiando as ações curativas, e a Enfermagem, fundamentalmente, se ateve ao atendimento biologicista, à prática hospitalocêntrica, destacando-se a realização de atividades administrativas e a supervisão dos integrantes da equipe de enfermagem. ${ }^{2} \mathrm{~A}$ enfermagem, como profissão, delega o monopólio do saber para o enfermeiro, mas a prática é desenvolvida por trabalhadores com diferentes níveis de formação. No entanto, o saber do enfermeiro não está isento da suposta dependência teórica das práticas científicas da área da saúde. ${ }^{4}$

Nos Estados Unidos, por volta de 1953, houve um movimento para a ampliação da cientificidade na Enfermagem, o que aconteceu com a construção de referenciais teóricometodológicos para a pesquisa e para a prática da Enfermagem. ${ }^{2}$ Esses referenciais foram importados pelas enfermeiras brasileiras e corroboravam a criação de novas escolas de graduação e da pós-graduação na modalidade de especialização. Na década de 1960 criaram-se escolas para a formação do técnico de enfermagem, cuja equipe passou a ser composta por enfermeiro, técnico, auxiliar e parteira, evidenciando a divisão técnica do trabalho na Enfermagem. A formação profissional exigia uma adequação aos desempenhos típicos da organização hierarquizada e da divisão do trabalho em um modelo curativo e cada vez mais especializado. ${ }^{3}$

Nas décadas subsequentes, o enfermeiro continuou consolidando seu saber e também seu poder sobre os demais membros da equipe de enfermagem, reafirmou-se como profissional de nível superior e desenvolveu uma prática com forte ênfase no gerenciamento do espaço assistencial e do processo de trabalho da equipe. ${ }^{5-6} \mathrm{Na}$ Enfermagem brasileira destaca-se, ainda, a partir dos trabalhos desenvolvidos por Wanda de Aguiar Hor ta em 1971 e da criação dos cursos de pós-graduação stricto sensu, a construção de um corpo específico de conhecimentos constituindo uma Teoria de Enfermagem, sinalizando novas perspectivas para o fazer desta profissão, a partir da Sistematização da Assistência de Enfermagem (SAE). ${ }^{7}$ A partir de 1998, a Sistematização da Assistência de Enfermagem constitui-se em um requisito legal para o desenvolvimento da prática assistencial, que foi reforçado com a aprovação de resoluções sobre o tema pelo Conselho Federal de Enfermagem (Cofen) - Resolução Cofen 272/2002, 
posteriormente atualizada e revogada pela Resolução Cofen 358/2009. ${ }^{8}$ A Sistematização da Assistência de Enfermagem ${ }^{7}$ compreende uma dinâmica de ações profissionais sistematizadas e inter-relacionadas, que visam ao aprimoramento técnico-científico e ao respaldo ético-legal para, desta maneira, fortalecer o vínculo profissional-cliente.

A sistematização traz em si o planejamento da assistência de enfermagem e tem o intuito de consolidar a enfermagem como profissão autônoma. ${ }^{9}$ Os referenciais teóricos que fundamentam esta sistematização centram-se em um conjunto de conceitos e afirmações relacionadas com visão aprofundada sobre o fenômeno a que se aplica o cuidado a pessoas. As Teorias de Enfermagem consistem em base científica para o fazer da enfermagem com qualificação, desenvolvendo cuidados terapêuticos com base em saberes próprios. $^{10}$

Com base na trajetória da Enfermagem para a constituição desses saberes, no plano pedagógico, assistencial e da produção científica, não deixaremos de considerar os prejuízos indesejáveis pela falta de poder como área de conhecimento, face às pressões das políticas de saúde ou pelas instâncias reguladoras dos órgãos de fomento e amparo à pesquisa. ${ }^{4}$

Neste contexto, o presente estudo teve por objetivo realizar uma análise reflexiva da Enfermagem como profissão à luz da sociologia das profissões de Eliot Freidson, em que a evolução da Enfermagem, do cumprimento de tarefas à profissão de consulta e ciência aplicada, se estabelece. Nesta reflexão, a análise do seu credencialismo é fator preponderante para a autonomia da profissão. Destaca-se neste artigo o caráter profissional da Enfermagem nos aspectos de propriedade de um conhecimento espećfico, seu credencialismo e as perspectivas de autonomia que apresenta.

\section{APROXIMAÇÃO À REFLEXÃO}

No decorrer do desenvolvimento da Enfermagem na perspectiva da modernidade, a partir do final do século XVIII, o seu fazer não se sustentava em um conhecimento próprio, especializado, como ciência diferenciada. Com o Sistema Nightingale de Enfermagem Moderna, o "cuidado" constitui-se em centro de interesse objetivado em teoria e prática. Caracterizou-se, assim, o cuidado "de responsabilidade específica das Enfermeiras, compreendido e focalizado como objeto de estudo no plano da construção do conhecimento científico, portanto das investigações da área da enfermagem". 11:409

A Enfermagem, em uma análise da sua evolução como profissão, desenvolveu uma abordagem da saúde centrada no reconhecimento dos comportamentos humanos e com isto concede significados às experiências dos indivíduos doentes. A educação em Enfermagem, a partir de conceitos próprios e estabelecidos em interação com outras ciências, sociologia, psicologia, antropologia e a física, desenvolve conhecimento próprio quando centraliza o cuidado, não na assistência à doença, mas nas reações humanas e no contexto que acontecem.

As credenciais para que uma ocupação seja considerada uma profissão incluem a expertise, a autonomia e a capacidade de se autorregular. ${ }^{1}$ Assim, a construção de um corpo de conhecimento específico da Enfermagem vem sendo buscado para encontrar metodologias e suportes científicos, que fundamentem a prática com vistas à prestação de cuidados seguros e ao reconhecimento social da necessidade deste trabalho profissional. As teorias de enfermagem falam por si da inerência fundante, ou da internalidade epistemológica e da historicidade da enfermagem. ${ }^{6} 0$ conhecimento especializado e a busca pela autonomia profissional defendidos por Freidson articulam-se à necessidade da atividade política para potencializar o credencialismo da profissão. Isso se configura na promoção contínua de desenvolvimento de projetos e incentivos à pesquisa, para o aprimoramento do saber e da competência em enfermagem. Assim se sucede na luta políticoprofissional pela sua regulamentação, definindo campo específico de atuação, além de direitos e deveres para os seus exercentes.

Os espaços de cuidado e atenção à saúde diversificamse, e o domínio de conhecimentos sobre o cliente e a doença passa da visão de um profissional para o compartilhamento de múltiplas práticas com saberes específicos e sistematizados. A Enfermagem é uma das profissões de saúde que, no sentido de Freidson, caracterizam-se como "profissões de consulta", por oferecerem serviços para resolver problemas práticos, diferenciando-se das "profissões acadêmicas ou eruditas" que não têm esta preocupação. ${ }^{1: 12} 0$ trabalho profissional da Enfermagem especifica-se, no conjunto das ações de saúde, pela realização de práticas de cuidado. Estas, no Brasil, estão autorregulamentadas para serem desenvolvidas pela equipe de enfermagem sob responsabilidade e supervisão do enfermeiro. Ressaltam-se as consultas de enfermagem, que são exercidas somente pelo enfermeiro e que envolvem exame físico, avaliação da condição geral do cliente, contextualização da alteração em relação à saúde e orientação para ações preventivas, de restabelecimento e curativas. Ao credencialismo da Enfermagem acrescentam-se a formação espećifica por pares e a regulação do exercício profissional reconhecida pelo Estado e proposta pelas organizações representativas da profissão.

\section{O CREDENCIALISMO DA ENFERMAGEM COMO PROFISSÃO}

Ao consideramos as características elencadas por Eliot Freidson para estruturar uma ocupação em profissão, destacando-se a capacidade de autorregulação como prova da sua autonomia, não podemos deixar de lado as polêmicas em torno da autonomia do enfermeiro e, no escopo dessas polêmicas a autonomia para realizar pesquisas, diagnóstico de situações de clientes necessitando de cuidados específicos, 
prescrição de medidas terapêuticas e de coordenação e controle da ação profissional. ${ }^{4}$

A base da formação universitária e os currículos instituídos influenciam o comportamento dos profissionais em geral. Novos estudos, tecnologias de aprendizagem e apropriações curriculares são necessárias para qualificar a socialização dos conhecimentos com vistas a comportamentos mais adequados ao cuidado da sociedade. ${ }^{1}$ A formação social e técnica recebida nas universidades oportunizam a distinção do profissional de um leigo.

Assim, Freidson estabelece a educação como pontochave não só para o credenciamento, mas também para determinar condutas individuais, internamente à profissão, com núcleos comuns de conhecimento e atitudes. E o conjunto de legislações e normatizações, determinadas pelo Estado e pelos órgãos representativos das profissões (associações, conselhos), orientam o modelo de formação profissional. Este padrão é voltado para a prestação de um serviço à coletividade, assim como o controle da formação requerida pelo Estado e pela profissão. $^{12}$

A sustentação do argumento de que a Enfermagem se apresenta como profissão de consulta, faz-se a partir do conhecimento construído como ciência aplicada por meio das Teorias de Enfermagem e da Sistematização da Assistência de Enfermagem. Estes são conhecimentos aplicados à pratica profissional fundamentados na Lei do Exercício Profissional da Enfermagem, Resoluções do Conselho Federal de Enfermagem e Código de Ética profissional. A sistematização da assistência de enfermagem é entendida como um importante instrumento de trabalho, que desenha o fluxo de realização, a natureza do trabalho a ser efetuado e determina o profissional da enfermagem por ele responsável. Fundamenta-se em referenciais teórico-filosóficos que norteiam habilidades cognitivas, emocionais e conceituais para o aprimoramento da produção de cuidado. A Sistematização da Assistência de Enfermagem - SAE define técnicas, recursos materiais e humanos, procedimentos, estratégias, objetivos para a realização da assistência e o gerenciamento do processo de trabalho em enfermagem e saúde. ${ }^{10}$

Assim, a prática profissional do enfermeiro distinguese da dos demais membros da equipe (técnicos e auxiliares de enfermagem) de enfermagem em termos de sustentação teórico-filosófica no processo de cuidar, ensinar e pesquisar. Além disso, a legislação garante que os cuidados de enfermagem sejam de responsabilidade primeira e última do enfermeiro, devendo ser executados diretamente por ele ou sob a sua supervisão.

Essas credenciais não são suficientes para tornar a Enfermagem uma profissão, entendendo-a como uma paraprofissão por não ter autonomia plena. Eénesta perspectiva que esta reflexão se faz pertinente. ${ }^{1}$

De que forma uma profissão pode ter autonomia plena uma vez que as práticas assistenciais, atualmente, se desenham em integração e em interdependência, profissão/profissões da saúde, profissão/sociedade? Dependem de legislações próprias e de controle do Estado no estabelecimento do monopólio sobre o seu saber e sobre a sua prática? As práticas de saúde integram variadas relações organizadas, o que distancia a assistência da clientela de práticas individuais plenamente autônomas. ${ }^{1}$

Percebe-se, então, que, a partir da cooperação profissional, a autonomia plena requerida como credencial para uma ocupação constituir-se uma profissão deixa de ter fundamento, uma vez que a complexidade da assistência em saúde requer o compartilhamento de decisões, de ações pensadas e de respeito aos espaços interprofissionais. A crítica, neste sentido, está em que, para a atenção em saúde, não cabe o domínio de uma profissão sobre outra, mas o respeito aos espaços próprios e inter-relação nos espaços comuns de atuação.

A caracterização da autonomia da enfermagem, ao nosso ver, evidencia-se pela implementação dos conhecimentos construídos em teorias e em formas específicas de sistematizar a assistência de enfermagem e por sua autorregulação. As teorias de enfermagem ${ }^{13}$ fazem com que reflitamos criativamente a prática, propiciam o domínio da profissão sobre o seu processo de trabalho e contribuem para o rompimento com a visibilidade atribuída a enfermagem, de executora de tarefas complementares ao ato médico, que não realiza uma avaliação própria sobre seu objeto de trabalho com consequente projeto de intervenção.

Diante das considerações defendidas por Freidson, uma profissão alcança sua autonomia quando consegue ela própria exercer controle sobre normas e desempenhos dos seus membros profissionais.

"A autorregulação é o teste da autonomia profissional". Então, analisemos! Se a profissão de consulta mantém vínculo direto com a clientela e necessita primordialmente das credenciais que a qualificam para garantir a aplicação de seus conhecimentos para o aprimoramento de suas ações, a Enfermagem se identifica como profissão de consulta. No que diz respeito à autorregulação, o profissional enfermeiro está credenciado para as práticas de cuidado, aplicando conhecimentos específicos no seu fazer e reconhecido em legislação própria. A Lei do Exercício profissional da Enfermagem Nú 7.498/86, em seu "art.11", dispõe que o enfermeiro exerce todas as atividades de enfermagem, cabendo-lhe: Iprivativamente: c) planejamento, organização, coordenação, execução e avaliação dos serviços da assistência de Enfermagem; i) consulta de enfermagem; j) prescrição de enfermagem". ${ }^{8}$ Concede claramente ao enfermeiro o direito a consulta de enfermagem, bem como outras prerrogativas para esta ação. Outras normatizações indicam a autonomia que a Enfermagem apresenta na organização das suas ações, como a Sistematização da Assistência de Enfermagem definida na Resolução Cofen nÚ 358/2009, que no seu art. 3 refere que "0 processo de enfermagem deve estar baseado em um suporte 
teórico que oriente a coleta de dados, o estabelecimento de diagnósticos de enfermagem e o planejamento das ações ou intervenções de enfermagem, e que forneça a base para a avaliação dos resultados de enfermagem alcançados". ${ }^{8}$ Este suporte, fundamentado no conhecimento, amplia a ação dos enfermeiros e organiza as atividades de toda a equipe de enfermagem. A comunicação multiprofissional torna-se facilitada, o que potencializa a atenção individualizada à clientela.

A Enfermagem evidencia-se como uma ciência aplicada, já que utiliza conhecimentos da ciência básica para finalidades práticas. Apresenta ${ }^{14}$ conhecimentos substantivos que tem como centralidade o processo humano-universo-saúde concretizado em estruturas conceituais e teorias de enfermagem. Refere-se, assim, a interdependência das respostas humanas no processo saúde-doença, em meio ao domínio biológico, social, comportamental e cultural. Pelo caráter subjetivo da aplicação do conhecimento científico, ainda é frágil na prática da Enfermagem Brasileira o cuidado sistematizado. Diante desta efemeridade, em que o saber da Enfermagem está posto é que a autonomia da profissão não se consolida e a sociedade não a percebe ainda com o poder de controle de práticas de saúde que a especificam. A profissão de Enfermagem tem uma prática desenvolvida em ações de saúde por equipe historicamente assimétrica e diferenciada em sua formação, e corresponde à influência da organização profissional que a representa. Neste sentido, o credencialismo por meio da Lei do Exercício profissional impulsiona a Enfermagem à apropriação das funções que Ihe são atribuídas, traz visibilidade à Enfermagem quando a assistência à saúde está sob práticas sistematizadas. ${ }^{5}$

Neste sentido, qual a real autonomia da enfermagem? A real autonomia é aquela que advém de uma iniciativa tomada, ${ }^{15}$ e a capacidade de criação e o poder de decisão diante de situações imprevistas, mesmo que no âmbito organizacional exista uma previsão e prescrição geral para tais acontecimentos.

A autonomia na enfermagem sustenta-se nos seguintes aspectos: 1) perspectiva do seu objeto de trabalho;2) perspectiva do âmbito das relações profissionais intraequipe e multiprofissional; 3) perspectiva da relação institucional/gestão; 4) perspectiva dos âmbitos público e privado (inclua-se domicilial); 5) perspectiva das práticas hospitalares e da atenção básica à saúde; 6) perspectiva do ensino/formação/pesquisa; 7) perspectiva do credencialismo; 8) perspectiva político-econômica. Evidenciando as perspectivas em que as relações de trabalho da Enfermagem se estabelecem, tem-se a amplitude da relatividade da autonomia por ela usufruída. 0 trabalho profissional "de consulta":1186 está como aquele que se refere à "busca de soluções para os problemas concretos dos indivíduos." Assim, a real autonomia da Enfermagem está em suas práticas de cuidado cooperativo a pessoas que dependem do seu conhecimento e da sua habilidade específica para solução de problemas, por meio de suas técnicas e o uso compartilhado de tecnologias. Está na articulação do seu saber direcionada às suas práticas assistenciais, advindas da sua capacidade de tomar decisões, fundamentadas no conhecimento específico e subsidiado por legislação própria, com entidades representativas for tes no âmbito da sociedade. ${ }^{6} \mathrm{~A}$ autonomia profissional relativa da Enfermagem é aparente nas práticas assistenciais em que há a coparticipação ativa de outros núcleos profissionais, e que se diferenciam nos sistemas público e privado. A autonomia mais ampla pode ser constatada na enfermagem acadêmica, de formação e pesquisa, a qual define em ações, decisões e modos de ensinar/pesquisar diretrizes próprias.

A abordagem freidsoniana, revisitada nesta reflexão, auxilia a análise da Enfermagem como profissão que historicamente transformou os espaços de cuidado e que continuamente, a partir da experiência, amplia o domínio de saberes e práticas. Neste sentido, as prerrogativas para a Enfermagem ser considerada uma ciência aplicada e profissão está estabelecida na expertisee na autorregulação, que culmina no credencialismo qualificacional da profissão.

\section{CONSIDERAÇÕES FINAIS}

As reflexões sobre a Enfermagem como profissão no âmbito das características definidas pela sociologia das profissões, na abordagem de Eliot Freidson, mostram-se ainda insipientes. No entanto, as discussões sociológicas no âmbito da Enfermagem vem ampliando-se e tem resgatado o seu entendimento como trabalho do campo da saúde, disciplina do conhecimento científico e como uma profissão orientada para o serviço. ${ }^{5}$

A partir da reflexão aqui realizada, consideramos que a Enfermagem é uma profissão de saúde. No Brasil, os membros da profissão responsabilizam-se por uma gama de fazeres fundamentados em conhecimentos científicos produzidos pelos pares. Os exercentes da profissão estão definidos legalmente e necessitam de formação específica, assim como têm suas atribuições e campo de atuação definidos em lei e reconhecidos pelo Estado e sociedade. 0 Estado reconhece o credenciamento formal de proteção aos membros da profissão. Esse reconhecimento é fundamental por tratarse de um trabalho que tem importância significativa na assistência em saúde, envolvendo o desenvolvimento de práticas baseadas em conhecimentos sistematizados, necessários para a permanente qualificação do cuidado.

Para além do cuidado dispensado em consulta e nas práticas diversas de cuidado há um corpo de conhecimentos que fundamenta a prática, fortalece o fazer técnico e contribui para a aproximação entre o profissional enfermeiro e a sociedade. No entanto, é necessário, ainda, convencer esta sociedade da sua utilidade enquanto profissão da área da saúde voltada às pessoas, à comunidade. ${ }^{12}$

A expertise e a autorregulação, que credenciam a Enfermagem como profissão, realizam o movimento necessário ao domínio dos espaços de trabalho em uma perspectiva do cuidado integral, equânime, interdisciplinar e multiprofissional. 
Os autores desta reflexão consideram que a autonomia profissional não deve ser reconhecida como liberdade do profissional para promover a subserviência de outros profissionais e, principalmente, daqueles que necessitam de assistência em saúde.

\section{REFERÊNCIAS}

1. Freidson E. Profissão médica: um estudo de sociologia do conhecimento aplicado. São Paulo: Editora UNESP; 2009.

2. Bock LF, Vaghetti HH, Bellaguarda MLR, Padilha MI, Borenstein MS. A organização da enfermagem e da saúde no contexto da idade contemporânea (1930-1960). In: Maria Itayra Padilha, Miriam S. Borenstein, Iraci dos Santos. Enfermagem história de uma profissão. São Caetano do Sul: Difusão Editora; 2011. p. 253-94.

3. Pires D. Hegemonia médica na saúde e a enfermagem. São Paulo: Cortez; 1989.

4. Carvalho V. Acerca da interdisciplinaridade: aspectos epistemológicos e implicações para a enfermagem. Rev. Esc. Enferm. USP. 2007 set.; 41(3): 500-7.

5. Kletemberg D, Vieira M, Bertoncini JH, Padilha MI, Borenstein MS. 0 fascínio da ciência na área da saúde (1960-1990). In: Maria Itayra Padilha, Miriam S. Borenstein, Iraci dos Santos. Enfermagem história de uma profissão. São Caetano do Sul(SP) : Difusão Editora; 2011. p. 254-95.

6. Pires D. A enfermagem enquanto disciplina, profissão e trabalho. REBEN. 2009 set./out.; 62(5): 739-44.

7. Barros ALBL, Lopes JL. A legislação e a sistematização da assistência de Enfermagem. Enferm. foco (Brasília). 2010; 1(2): 63-5.

8. Resolução 358, de 15 de outubro de 2009. Dispõe sobre a Sistematização da Assistência de Enfermagem (SAE). [Acessado em 2011 26 ago]. Disponível em: <http://www.site.portalcofen.gov.br/node/ 4384>.

9. Tannure MC, Pinheiro AM. SAE: Sistematização da Assistência de Enfermagem. Guia Prático. 2. ed. Rio de Janeiro: Guanabara Koogan; 2010.

10. Leopardi MT. Teoria e método em assistência de enfermagem. 2. ed. Florianópolis(SC): Ed. Soldasoft; 2006.

11. Carvalho V. Por uma epistemologia do cuidado de enfermagem e a formação dos sujeitos do conhecimento na área da enfermagem. Esc. Anna Nery Rev Enferm. 2009 jun; 13(2): 406-14.

12. Pereira Neto AF. Interfaces da história da Enfermagem: uma potencial agenda de pesquisa. Esc. Anna Nery Rev. Enferm. [periódicos na internet]. 2006 Dez [citado em 12 dez 2011]; 10(3). Disponível em: <http:// www.scielo.br/scielo.php?script =sci_arttext\&pid $=S 1414$ 1452006000300023 \&lng $=$ en\&nrm=iso >.
13. Pires D. Construir um novo saber de enfermagem: um dos nossos desafios para a virada do século. In: Anais do $42^{\circ}$ Congresso Brasileiro de Enfermagem; 1999 Out 11-16 Natal, Brasil. Natal(RN): Associação Brasileira de Enfermagem; 1990. p. 69-75.

14. Barret EAM. What is nursing science? Nurs Sci Q. 2002 jan.; 15(1): 51-60.

15. Rosenfield CL. Autonomia outorgada e relação com o trabalho: liberdade e resistência no trabalho na indústria de processo. Sociologias 2003 Jul./Dez; 5(10): 350-78. 